\title{
Biobank provides leads for selecting guide dogs
}

TOKYO

Biobanks, which combine genetic and lifestyle data from thousands of people, are increasingly being used to help researchers understand the roots of disease. But they can be used to study other things, such as behaviour. An experimental biobank set to open in Japan next month aims to do just that - for dogs.

Four years ago Hiroshi Suzuki, a professor of functional genomics at the Obihiro University of Agriculture and Veterinary Medicine in Hokkaido, found out that Japan suffers from an acute shortage of guide dogs, and decided to do something about it. Training guide dogs is time-consuming and expensive, costing about $¥ 2.7$ million (US\$23,000) per

dog. And even among labrador retrievers, which are particularly suited to the role, only about $30 \%$ of the dogs that are trained work out as guide dogs. Unfortunately, it is difficult to predict which puppies will make good guide dogs, says Takafumi Wada, head of the guide-dog association in Hokkaido, with which Suzuki is collaborating. And that's where Suzuki's database comes in.

Guide dogs have to be neutered before training starts, so successful dogs cannot be used for breeding. Suzuki gets round this by freezing testis and ovary tissue from dogs before training, with a view to breeding from the best. But ultimately he wants to develop genetic tests to select suitable dogs for breeding and to measure puppies' aptitude before they enter training. He adds that a similar approach could be applied to select dogs suitable for police duties or to make good pets.

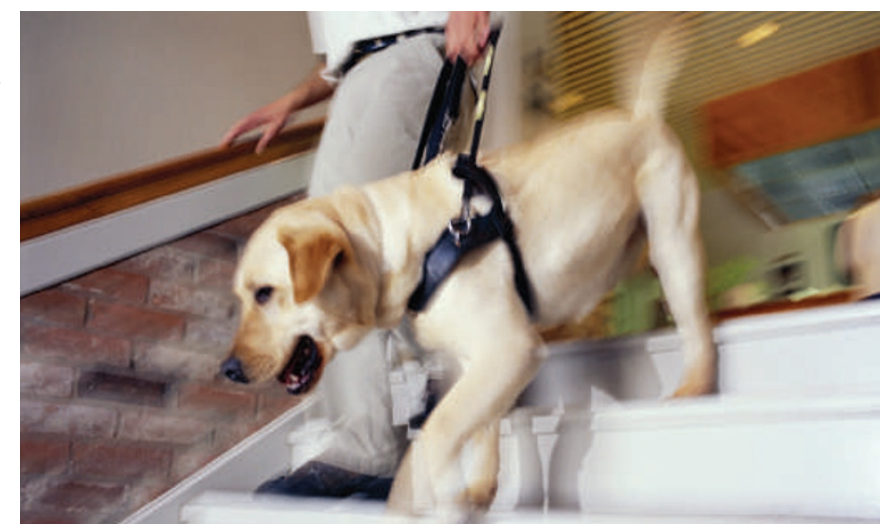

So far, Suzuki has screened DNA from 200 labradors. He has looked at 13 genetic variations called single nucleotide polymorphisms, or SNPs, and found five SNPs in three genes that he believes are related to guide-dog ability. In particular, a gene that codes for an enzyme called COMT (catechol-O-methyltransferase), which partly regulates mood in humans, seems to be involved in dogs' ability to concentrate, he says. In Suzuki's study, $80 \%$ of dogs with all five SNPs passed their training, whereas the pass rate was only $60 \%$ for dogs with just one of the SNPs. Suzuki is now writing up his results for publication.

Suzuki admits that the number of dogs he has studied so far is too small to confirm the role of these genes in determining guidedog ability, and he is working with guide-dog organizations in Finland and South Korea to collect at least 1,000 blood samples.

"Suzuki has a very innovative approach," says Yoshihisa Yamane, head of the Japan Veterinary Medical Association in Tokyo. "We hope he'll be able to breed more successful guide dogs."

But Masanari Itokawa, who studies schizophrenia at the Tokyo Institute of Psychiatry, is sceptical, pointing out that the link between genetics and behaviour tends to be complex, and that large-scale studies using thousands of SNPs will be needed. "We can't find the relations between gene mutations and personalities or behaviours so easily," he says.

But Suzuki thinks his work, which can draw on the dogs' detailed family histories, could serve as a model for human disease studies. Ichiko Fuyuno

Lab work: a canine biobank aims to ease Japan's shortage of guide dogs.

\section{SCORECARD}

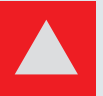

Chinese pest control Shanghai residents are being encouraged to breed fish that feed on mosquito larvae as an environmentally friendly way to combat this summer's expected plague of

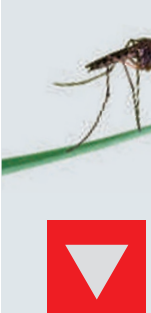
the insects.

the International Space Station after astronaut Sunita Williams accidentally sprayed the cabin with it while treating herself

to some zero-gravity sushi.

ON THE RECORD

'CThere's a trend but the error is big (increase the $N$ ),

\section{The rat's no good, use the guinea pig (increase the $\boldsymbol{N}$ ), \\ The antibody's weak, the background high (increase the $N$ ), My P value is bigger than pi (increase the $\boldsymbol{N}$ )")}

Lyrics to Increase the $N$, a Ramones-esque tribute to science by Canadian rockers HEFE. To hear it, visit www.myspace.com/heferocks

\section{WORDWATCH}

Conservapedia: The new conservative encyclopaedia (www.conservapedia.com) that allows conservative Christians to hit back at the 'inherent liberal bias' of Wikipedia. Under 'Global warming', for example, it warns us that "scientists are mostly liberal atheists, untroubled by the hubris that man can destroy the Earth which God gave him".

Sources: Reuters, Associated Press, The World's Fair 